\title{
INVESTIGACIÓN EN LA FACULTAD DE COMUNICACIÓN. DEPARTAMENTO DE SOCIOLOGÍA
}

Mikel Altuna (Universidad de Sevilla)

http://dx.doi.org/10.12795/AdMIRA.2009.01.19

Las diferentes parcelas o áreas en las que se divide el conocimiento en muchas ocasiones presentan dificultades para ser definidas o caracterizadas por la especificad del objeto o campo en el que se centran. En otras ocasiones, es igualmente difícil observar las diferencias en las herramientas metodológicas y en las técnicas utilizadas por las aproximaciones de diferentes áreas de conocimiento. Lo cierto es que en el campo de las Ciencias Sociales son muchas las interrelaciones, las complementariedades y por tanto, son muy necesarias las colaboraciones entre las diferentes áreas, propiciando así la compaginación de los diferentes acentos particulares para conseguir dar mejor cuenta de una realidad compleja. Este es claramente el caso de la Comunicación. Las divisiones reales existentes en cuanto a las parcelas del conocimiento tienen que ver lógicamente con los diferentes procesos sociales de su institucionalización académica y lo que debe primar para el futuro es la colaboración y complementariedad interdepartamental.

Desde los orígenes de la investigación social, desde la consolidación de la sociología como ciencia y desde su institucionalización académica, la Comunicación ha sido uno de los campos en los que se ha centrado su particular perspectiva, bien en investigaciones realizadas por los propios sociólogos o por otros investigadores afines que utilizan su acervo teórico-metodológico.

El Departamento de Sociología de la Universidad de Sevilla tiene una vida propia, como departamento independiente, de casi la misma temporalidad que la Facultad de Comunicación, contemplada también en el proceso temporal con su anterior denominación. Sin contar con una facultad específica de Sociología en la Universidad Hispalense, los aproximadamente 25 docentes del Departamento imparten su docencia e investigan en siete facultades, incluida la de Comunicación, que siempre ha sido una de las más importantes para el Departamento. Sus campos de actividad e investigación son por lo tanto amplios y diversos y los recursos limitados. El profesorado de la Facultad de Comunicación se ha compuesto como promedio anual por siete personas que trabajan e investigan sobre Comunicación pero igualmente sobre otras parcelas del conocimiento. De igual manera miembros del Departamento adscritos a otros centros también han trabajado sobre Comunicación. 
En el presente recopilatorio solamente recogeremos en sus líneas principales los grupos de investigación, los proyectos de investigación y las publicaciones más recientes y relevantes en el “campo de la Comunicación”, en las que participan miembros del Departamento con docencia y adscripción a la Facultad de Comunicación. Campo de la Comunicación difícil de delimitar y en el que coinciden diversas perspectivas que caracterizan la necesidad y pertinencia de estudios interdisciplinares e interdepartamentales para poder dar cuenta mejor de una realidad poliédrica.

\section{GRUPOS DE INVESTIGACIÓN}

Se mencionan exclusivamente los componentes y los proyectos en curso (2008) sin contar las ayudas a la Consolidación de Grupos de Investigación.

\section{Análisis de las Estructuras de Interactividad e Intercomunicación Social (SEJ029)}

Responsable: $\quad$ Eduardo Bericat Alastuey

Componentes: Manuel Jesús Caro Cabrera), Julia Espinosa Fajardo, Manuel Herrera Usagre, María Luisa Jiménez Rodrigo, Elena Marín Cassinello, Esther Márquez Lepe, María Dolores Martin-Lagos López, $\quad$ Eva Sánchez Bermejo.

Ayudas/Contratos/Proyectos gestionados por la USE en los que participan miembros de este Grupo en el Departamento:

-Estudio y análisis de los valores sociales (68/83) - Fundación Centro de Estudios Andaluces: 43.305,73€. Integrantes: Eduardo Bericat Alastuey (Resp.)

-Puesta en marcha del nuevo sistema de indicadores sociales de Andalucía y elaboración de un análisis de la situación social de la población andaluza (68/83). Tiene como objetivo crear un sistema de indicadores sociales que permita monitorizar la evolución de la sociedad andaluza. Vigencia: 2008-2009 - Instituto de Estadística de Andalucía: 152.905,74 €. Integrantes: Eduardo Bericat Alastuey (Resp.), Miguel Ángel Altuna Isasmendi, María de las Mercedes Camarero Rioja, Manuel Herrera Usagre, María Luisa Jiménez Rodrigo, Elena Marín Cassinello, Eva Sánchez Bermejo, José Juan Venteo de Haro 
-Revisión del marco teórico del sistema de indicadores sociales de Andalucía (68/83) - Instituto de Estadística de Andalucía: 83.542,53€. Integrantes: Eduardo Bericat Alastuey (Resp.), Miguel Ángel Altuna Isasmendi, María de las Mercedes Camarero Rioja, José Juan Venteo de Haro

\section{Tes (Trabajo, Eco-Economia y Sociedad) (SEJ335)}

Grupo Interdepartamental con el Dpto. Comunicación Audiovisual, Publicidad y Literatura. Responsable: Lina Gavira Álvarez

Componentes: Maria de Arcos Rus, Cristobal Gómez Benito), Francisco González Turmo, Daniel López García, Pilar Ortega Espejo, Jacinto Manuel Porro Gutierrez

\section{PRINCIPALES LINEAS DE INVESTIGACIÓN EN COMUNICACIÓN Y PUBLICACIONES DE LOS RESPONSABLES DE LOS GRUPOS DE INVESTIGACIÓN}

-EDUARDO BERICAT ALASTUEY, Catedrático de Sociología, adscrito al Departamento de Sociología de la Universidad de Sevilla, y profesor de la Facultad de Comunicación, ha realizado diversas investigaciones sociales en las que se consideran especialmente la dimensión comunicativa de la realidad social. Desde la perspectiva de la sociología de las emociones, ha aplicado el Análisis de Contenido Emocional a las unidades expresivas publicadas por los medios de comunicación. También ha trabajado la relación metodológica y práctica existente entre el trabajo periodístico y el sociológico (Socioperiodismo). En la actualidad investiga en el campo de la Sociología Visual, analizando la dialéctica significativa existente entre texto e imagen, así como entre imagen y conocimiento social. Es responsable de un grupo de investigación dedicado al Análisis de Estructuras de Interactividad e Intercomunicación Social.

BERICAT ALASTUEY, E., (2005): "La cultura del horror en las sociedades avanzadas: de la sociedad centrípeta a la sociedad centrífuga”, en Revista Española de Investigaciones Sociológicas, REIS, $n^{\circ} 110$, pp. 53-89. 
BERICAT ALASTUEY, E., (2002): “¿Sienten las sociedades? Emociones individuales, sociales y colectivas”, en P. Fernández y N. Ramos (coord.), Corazones inteligentes, pp. 121-144 , Barcelona, Kairós.

UBERICAT ALASTUEY, E., (2002): "Rituales mediáticos, emociones colectivas y orden social”, en VV AA, La sociedad: Teoría e Investigación empírica. Libro homenaje a José Jiménez Blanco, Madrid, Centro de Investigaciones Sociológicas, CIS, pp.765-782.

BERICAT ALASTUEY, E., (1996): "La sociedad de la información: Tecnología, cultura y sociedad” en Revista Española de Investigaciones Sociológicas, REIS n o 76, pp. 99-121.

BERICAT ALASTUEY, E., (1999): "El contenido emocional de la comunicación en la sociedad del riesgo. Microanálisis del discurso", en Revista Española de Investigaciones Sociológicas, REIS nº 87, pp.221-253.

(1995) BERICAT ALASTUEY, E. (Dir. y Coord.), Una ciudad al sur. Documentos socioperiodísticos de la ciudad de Málaga, Málaga, Universidad de Málaga.

(1995) BERICAT ALASTUEY, E., “¿Qué es un documento socioperiodístico?”, en E. Bericat (Dir. Y Coord.), Una ciudad al sur. Documentos socioperiodísticos de la ciudad de Málaga, Málaga, Universidad de Málaga.

LINA GAVIRA ÁLVAREZ, Profesora Titular de Universidad en la Facultad de Comunicación de la Universidad de Sevilla, Vocal del Comité Andaluz para la Sociedad del Conocimiento del Parlamento de Andalucía, consultora y experta de diferentes instituciones de la UE y del Consejo de Europa.

\section{LÍNEAS DE TRABAJO DESARROLLADAS EN LA INVESTIGACIÓN SOBRE COMUNICACIÓN.}

- Papel de la comunicación en la promoción de la sociedad del conocimiento.

- Comunicación, conocimiento y alimentación buena, limpia y justa.

- Desarrollo local y el papel de la comunicación horizontal y vertical en las organizaciones.

- Nuevas tecnologías de la información, especialmente de grupos vulnerables. 
- Necesidades y acceso de los jóvenes a la información y el asesoramiento.

- Nuevas formas de comunicación y coordinación de los servicios de empleo y de política social;

- Redes de comunicación y acceso al autoempleo para mujeres y grupos vulnerables;

- Redes sociales y comunicación para el Empleo, las políticas de desarrollo y protección social en zonas rurales.

- La comunicación en las nuevas formas de gestión del empleo para grupos especialmente desfavorecidos: Empresas de inserción social.

- Creación y desarrollo de Nuevas herramientas audiovisuales de motivación y asesoramiento para el empleo y la inclusión social de grupos vulnerables y desfavorecidos:

- Ministerio de Trabajo y Asuntos Sociales (jóvenes: Muévete) (1997-1998)

- Instituto Andaluz de la Mujer (mujeres: Hazte valer) (1999-2000)

- Creación e implementación de sistema de comunicación-formación-gestión territorializado para la creación de empresas sostenibles a nivel local de mujeres y grupos desfavorecidos:

- Red local para la mejora de condiciones de vida y trabajo (1995-1998)

- Red local FLIIPAT para inclusión sociolaboral de inmigrantes en Sevilla (2005)

- Formación-acción a nivel local para mujeres formadoras y responsables políticas en ecotecnologías en Andalucía en partenariado con las redes de Alemania, Italia y Austria (Red Girls Ecology Action) (2002)

- Procesos innovadores de coordinación a nivel local de motivación-formaciónasesoramiento y creación de 5 empresas con mujeres desfavorecidas (1995-1998).

- PUBLICACIONES CIENTÍFICAS Y DE DIFUSIÓN VINCULADAS CON LA COMUNICACIÓN.

Gavira, L (2008) "Políticas de inclusión en el marco de la reestructuración de las administraciones públicas" en Inserción social y derechos humanos. Diputación Provincial de Córdoba y Asociación de DDHH de Andalucía. 
Gavira, L \& González, F. (2004) Necesidades de información y asesoramiento de los jóvenes en Andalucía. Estrategias y buenas prácticas. Instituto Andaluz de la Juventud. Consejería de Presidencia de la Junta de Andalucía. Sevilla. 394 pág. ISBN 84-87632-11-4

Gavira, Lina (2004) Digitalización de la sociedad y gobernanza: el papel de las nuevas tecnologías de la información y la comunicación en el acceso de los ciudadanos a los servicios. Boletín de la asociación latinoamericana de investigación de la comunicación. año iv, bol. 17. junio-julio 2004. www.eca.usp.br/alaic/boletin17/lina

\section{INFORMES Y PROYECTOS RECIENTES Y RELEVANTES}

Gavira, L. \& González, F. (2004) The role of new information technology as regards user involvement in social services. Council of Europe. Integrated Proyect 1 Grupo de trabajo Making democratic institutions work.www.coe.int/t/e/social_cohesion/social_policies.

Gavira, L. (Directora Y Coordinadora del proyecto) "HAZTE VALER" Video y manual de uso de la herramienta audiovisual de motivación y orientación para la inserción sociolaboral de mujeres jóvenes. GEISE, S.L., ISIS Producciones, F. González, J. Blanco y A. Caro. Financiación: Instituto Andaluz de la Mujer de la Junta de Andalucía. (2000). (Producido a partir del proyecto: “Investigación cualitativa aplicada a la motivación y orientación para la inserción sociolaboral de jóvenes con especiales dificultades a través de la metáfora iconográfica, con técnica de representación socio dramática).

Gavira, Lina (1998) Guión del video sobre resultados del proyecto de inserción sociolaboral nacido del proyecto de investigación-acción: “Asesoramiento para mujeres en países del sur de Europa. Now - Carmona". Video de difusión de resultados.

Gavira, L. (1998) “Muévete”. Manual de uso y herramienta audiovisual de orientación para jóvenes con especiales dificultades. Manual de 35 pag. y Vídeo (20’ de duración) para la motivación y orientación para el empleo. Fundación Trini Jove, Asociación los Alcores, GEISE, S.L. Ministerio de Trabajo y Asuntos Sociales. Madrid. (Producido a partir del proyecto de investigación: “Análisis cualitativo para la producción de una herramienta de socio dramatización audiovisual dirigida a la motivación y orientación para la inserción sociolaboral de jóvenes con especiales dificultades). 


\section{OTRAS PUBLICACIONES RELEVANTES RECIENTES}

Profesora Dra. Esther Márquez Lepe. Su tesis y diversas publicaciones tienen una relación directa con el análisis del discurso político, concretamente, parlamentario. Las dos publicaciones más recientes sobre este último ámbito son:

Márquez Lepe, Esther (2008). Evolución histórica de los discursos políticos sobre inmigración en España: representaciones y contextos. En Checa, F (ed) La inmigración sale a la calle. Comunicación y discursos políticos sobre el fenómeno migratorio.Barcelona: Icaria Antrazyt.

Marquez Lepe, Esther (2007). La gestión parlamentaria del discurso político sobre inmigración en España. En Zapata Barrero, R y Van Dijk, T (eds). Discursos sobre la inmigración en España. Los medios de comunicación, los parlamentos y las administraciones. Barcelona: Fundación CIDOB. 